\title{
miR-503 regulates the resistance of non-small cell lung cancer cells to cisplatin by targeting Bcl-2
}

\author{
TIANZHU QIU ${ }^{1 *}$, LI ZHOU $^{1 *}$, TONGSHAN WANG ${ }^{1}$, JING XU $^{1}$, JIAN WANG $^{1}$, WENJIAO CHEN ${ }^{1}$, \\ XIN ZHOU ${ }^{1}$, ZEBO HUANG ${ }^{1}$, WEI ZHU ${ }^{1}$, YONGQIAN SHU ${ }^{1,2}$ and PING LIU ${ }^{1,2}$ \\ ${ }^{1}$ Department of Oncology, The First Affiliated Hospital of Nanjing Medical University, \\ ${ }^{2}$ Cancer Center of Nanjing Medical University, Nanjing, Jiangsu 210029, P.R. China
}

Received February 27, 2013; Accepted May 16, 2013

DOI: $10.3892 /$ ijmm.2013.1439

\begin{abstract}
Drug resistance is one of the leading causes of chemotherapy failure in cancer treatment. MicroRNAs (miRNAs or miRs) are short non-coding RNA molecules that post-transcriptionally regulate gene expression and play a critical role in diverse biological processes. In this study, we report that miR-503 regulates the resistance of non-small cell lung cancer cells to cisplatin. The expression of miR-503 was decreased in the cisplatin-resistant non-small cell lung cancer cells, A549/CDDP, compared with the parental A549 cells. The overexpression of miR-503 sensitized the A549/CDDP cells to cisplatin, whereas the inhibition of miR-503 in the A549 cells increased resistance to cisplatin. Mechanistically, miR-503 specifically targeted Bcl-2, an anti-apoptotic protein upregulated in the A549/CDDP cells. The ectopic expression of miR-503 reduced the Bcl-2 protein level and sensitized the A549/CDDP cells to cisplatin-induced apoptosis. Taken together, our results suggest that miR-503 regulates cell apoptosis, at least in part by targeting $\mathrm{Bcl}-2$, and thus modulates the resistance of non-small cell lung cancer cells to cisplatin.
\end{abstract}

\section{Introduction}

Lung cancer, predominantly non-small cell lung cancer, is the leading cause of cancer-related mortality worldwide. Patients with non-small cell lung cancer are mostly treated with platinum-based chemotherapy, often in combination with radiation therapy. However, the development of chemoresistance, either intrinsic or acquired, is a major obstacle limiting

Correspondence to: Professor Ping Liu or Dr Wei Zhu, Department of Oncology, The First Affiliated Hospital of Nanjing Medical University, 300 Guangzhou Road, Nanjing, Jiangsu 210029, P.R. China

E-mail: liu-ping@csco.org.cn

E-mail: belliwether@163.com

*Contributed equally

Key words: miR-503, cisplatin resistance, apoptosis, Bcl-2, lung cancer successful treatment (1). Previous studies have indicated the cytological mechanisms of drug resistance in cancer cells, such as increased detoxification of anticancer drugs by the glutathione system, a defective apoptotic pathway, enhanced DNA repair or increased tolerance to DNA damage, decreased uptake of water-soluble drugs and enhanced drug efflux from cancer cells mediated by ATP-binding cassette (ABC) transporters (1-4). Studies have shown that epigenetic changes, including aberrant $\mathrm{CpG}$ island methylation, histone modifications and abnormal expression of microRNAs (miRNAs or miRs) may be responsible for the drug resistance of cancer cells. Specifically, epigenetic changes as opposed to genetic mutations may play a crucial role in the acquired drug resistance of cancer cells (5-7). Among these, miRNAs, as a class of small non-coding RNAs of 18-24 nucleotides, are posttranscriptional regulators that bind complementary sequences of target mRNAs, usually resulting in translational repression or target degradation and gene silencing (8). By regulating gene expression at the post-transcriptional level, miRNAs have been linked to pathways associated with cell differentiation, proliferation and survival, and their aberrant expression has been shown to be involved in drug resistance in different types of tumors (9), such as breast cancer, colorectal carcinoma, gastric cancer and glioblastoma multiforme (10-15).

Recently, there has been increasing interest in understanding the role of miR-503 in cancer. miR-503 has been shown to be downregulated in oral cancer cells and hepatocellular carcinoma cells $(16,17)$, whereas it is overexpressed in parathyroid carcinoma, retinoblastoma and adrenocortical carcinoma (18-20). The upregulation of miR-503 is associated with a shorter overall survival rate among patients with adrenocortical carcinoma (18). A previous study demeonstrated that miR-503 is a novel regulator of RBP CUG-binding protein 1 (CUGBP1) expression, and thus increases the sensitivity of intestinal epithelial cells to apoptosis (21). Moreover, miR-503 has been shown to reduce the $\mathrm{S}$ phase cell population and to inhibit cell growth by suppressing endogenous CCND1 at the protein and mRNA level, suggesting that miR-503 is a putative tumor suppressor (22). miR-503 has also been shown to induce G1 phase arrest and to inhibit the migration and invasion of HCCLM3 hepatocellular carcinoma cells in vitro (16). The altered expression of miR-503 affects the immunity response to radiotherapy and is a negative regulator of CD40 in irradiated 
U937 cells (23). However, little is known about the effects of miR-503 in the development of drug resistance in lung cancer.

In this study, we demonstrate that miR-503 is downregulated in the drug-resistant A549/CDDP human non-small cell lung cancer cell line, compared with the parental A549 cell line. Our results suggest that miR-503 plays a role in the development of drug resistance in human non-small cell lung cancer cells, at least in part by targeting the anti-apoptotic protein, Bcl-2.

\section{Materials and methods}

Cell culture. The A549 human lung cancer cell line and its drug-resistant variant, A549/CDDP (both obtained from Biosis Biotechnology Co., Ltd., Shanghai, China), were cultured in RPMI-1640 medium supplemented with $10 \%$ fetal calf serum (Gibco-BRL, Grand Island, NY, USA) in a humidified atmosphere containing $5 \% \mathrm{CO}_{2}$ at $37^{\circ} \mathrm{C}$. To maintain the drug-resistant phenotype, cisplatin (at a final concentration of $4 \mu \mathrm{g} / \mathrm{ml}$ ) was added to the culture medium for the A549/ CDDP cells.

Real-time PCR analysis for miRNA. Total RNA from the A549 and A549/CDDP cells was isolated using TRIzol reagent (Invitrogen, Carlsbad, CA, USA) and the miRNA fraction was further purified using a mirVana ${ }^{\mathrm{TM}}$ miRNA isolation kit (Ambion, Austin, TX, USA). The concentration and purity of the RNA samples were determined spectroscopically. Realtime reverse transcription PCR (qRT-PCR) was performed using the ViiA 7 Real-Time PCR System with SYBR-Green Master Mix. As a control, the small housekeeping U6 gene was amplified and quantified. The EzOmics ${ }^{\mathrm{TM}}$ miRNA qPCR Detection Primer Set (cat no. BK1010) and the EzOmics ${ }^{\mathrm{TM}}$ One-Step qPCR kit (cat no. BK2100), which were purchased from Biomics Biotechnologies Co., Ltd. (Nantong, China), were used for real-time PCR analysis for miR-503 and U6 snRNA, respectively (24). The fold-change for miRNA from the A549/ CDDP cells relative to the control A549 cells was calculated using the $2^{-\Delta \Delta \mathrm{Ct}}$ method (25), where $\Delta \Delta \mathrm{Ct}=\Delta \mathrm{Ct} \mathrm{A} 549 /$ $\mathrm{CDDP}-\Delta \mathrm{Ct} \mathrm{A} 549$ and $\Delta \mathrm{Ct}=\mathrm{Ct}$ miRNA $-\mathrm{Ct} \mathrm{U} 6$ small nuclear RNA (snRNA). PCR was performed in triplicate.

In vitro drug sensitivity assay. The A549/CDDP and A549 cells were plated in 6-well plates $\left(6 \times 10^{5}\right.$ cells/well) and $100 \mathrm{nM}$ of the miR-503 mimic or $100 \mathrm{nM}$ miRNA mimic control were transfected into the A549/CDDP cells, while $100 \mathrm{nM}$ of the miR-503 inhibitor or $100 \mathrm{nM}$ miRNA inhibitor control were transfected into the A549 cells, using Lipofectamine-2000 (Invitrogen, Grand Island, NY, USA) according to the manufacturer's instructions The miR-503 mimic, miRNA mimic control, 2'-O-methyl (2'-O-Me) modified miR-503 inhibitor, and miRNA inhibitor control were chemically synthesized by Shanghai GenePharma Co., Ltd. (Shanghai, China).

Twenty-four hours after transfection, the cells were seeded in 96-well plates $\left(5 \times 10^{3}\right.$ cells/well) for the following experiment. After cellular adhesion, freshly prepared anticancer drug (cisplatin; Qilu Pharmaceutical Co., Ltd., Jiinan, China) was added at a final concentration of 0.01-, 0.1-, 1- and 10-fold higher than the human peak plasma concentration as previously described (24). The peak serum concentration was $2.0 \mu \mathrm{g} / \mathrm{ml}$ for cisplatin as previously described $(26,27)$. Forty-eight hours after the addition of the drug, cell viability was assessed by the 3-(4,5-dimethylthiazol-2-yl)-2,5-diphenyl-tetrazolium bromide (MTT) assay. The absorbance at $490 \mathrm{~nm}$ (A490) of each well was read on a spectrophotometer. The concentration at which the drug produced 50\% inhibition of growth (IC50) was estimated by the relative survival curve. Three independent experiments were performed in duplicate.

Dual luciferase activity assay. The 3'-UTR of human Bcl-2 cDNA containing the putative target site for miR-503 was chemically synthesized and inserted into the $X b a \mathrm{I}$ site, immediately downstream of the luciferase gene in the pGL3control vector (Promega, Madison, WI, USA) by Biomics Biotechnologies Co., Ltd. Twenty-four hours prior to transfection, the cells were plated at $1.5 \times 10^{5}$ cells/well in 24-well plates. Two hundred nanograms of pGL3-Bcl-2-3'-UTR plus $80 \mathrm{ng}$ pRL-TK (Promega) were transfected in combination with 60 pmol of the miR-503 mimic or miRNA mimic control using Lipofectamine 2000 (Invitrogen) according to the manufacturer's instructions as previously described (24). Luciferase activity was measured $24 \mathrm{~h}$ after transfection using the Dual Luciferase Reporter Assay System (Promega). Firefly luciferase activity was normalized to Renilla luciferase activity for each transfected well. Three independent experiments were performed in duplicate.

Western blot analysis. A549/CDDP cells were plated in 6-well plates $\left(6 \times 10^{5}\right.$ cells/well). Seventy-two hours after the transfection of the miR-503 mimic or miRNA mimic control, the cells were harvested and homogenized with lysis buffer. Total protein was separated by denaturing on 10\% SDS-polyacrylamide gel electrophoresis. Western blot analysis was performed as previously described (14). The primary antibodies against Bcl-2 and $\alpha$-tubulin were purchased from Cell Signaling Technology (Danvers, MA, USA) and Bioworld Technology (Minneapolis, MN, USA), respectively. Protein levels were normalized to $\alpha$-tubulin. Fold-changes were determined.

Apoptosis assay. Cells were plated in 6-well plates $\left(6 \times 10^{5}\right.$ cells/ well). Twenty-four hours after transfection, the A549/CDDP cells were treated with cisplatin at a final concentration of 5 and $20 \mu \mathrm{g} / \mathrm{ml}$. Forty-eight hours following treatment with cisplatin, flow cytometry was used to detect the apoptosis of the transfected A549/CDDP cells by determining the relative amount of Annexin V-FITC-positive-PI-negative cells as previously described (14).

Statistical analysis. Each experiment was repeated at least three times. Numerical data are presented as the means $\pm \mathrm{SD}$. The differences between means were analyzed using the Student's t-test. All statistical analyses were performed using SPSS 11.0 software (SPSS Inc., Chicago, IL, USA). A P-value $<0.01$ was considered to indicate a statistically significant difference.

\section{Results}

miR-503 is downregulated in A549/CDDP cells compared with A549 cells. To determine whether miR-503 is involved in the development of drug resistance in non-small cell lung cancer 


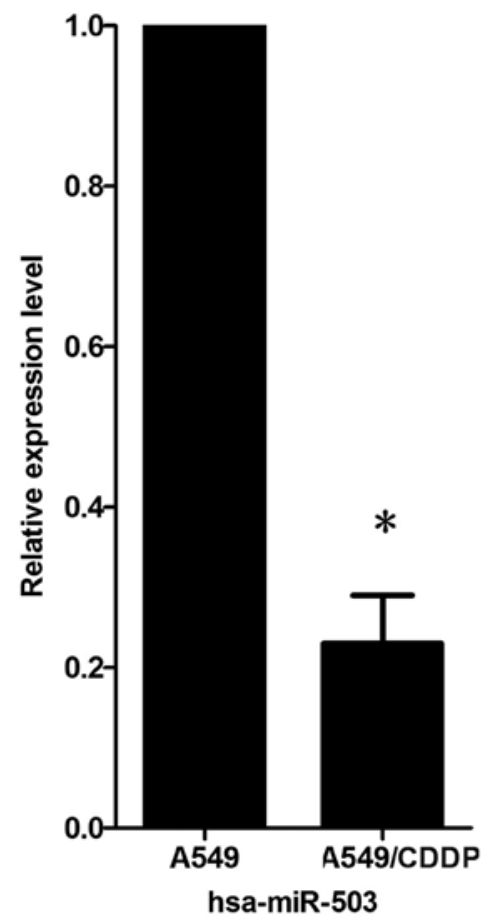

Figure 1. Real-time quantification of miR-503 by stem-loop reverse transcription and real-time PCR (RT-PCR) showed that miR-503 was downregulated in the A549/CDDP cisplatin (CDDP)-resistant cell line compared with the A549 cell line. Triplicate assays were performed for each RNA sample, and the relative amount of miR-503 was normalized to U6 snRNA. Data are presented as fold-changes of miR-503 levels in the A549/CDDP cells relative to the A549 cells, which was set as 1 (means $\pm \mathrm{SD}$ ). ${ }^{*} \mathrm{P}<0.01$.

cells, the level of miR-503 was analyzed in the A549/CDDP cells compared with their parent cell line, A549. Real-time PCR for miR-503 verified that miR-503 was significantly downregulated in the A549/CDDP cells compared with the parental cells, A549, and the decreased fold-change was $4.35 \pm 0.06$ (Fig. 1).

miR-503 regulates the resistance of human lung cancer cells to cisplatin. In the A549/CDDP cells, MTT assay revealed that the cells transfected with the miR-503 mimic exhibited significantly decreased resistance to cisplatin compared with the miRNA mimic control-transfected cells (Fig. 2A); whereas in the A549 cells, the cells transfected with the miR-503 inhibitor exhibited significantly enhanced resistance to cisplatin compared with the miRNA inhibitor control-transfected cells (Fig. 2B). These results suggest that miR-503 regulates the resistance of human lung cancer cells to cisplatin.

Anti-apoptotic Bcl-2 is the target gene of miR-503. TargetScan Human 5.1 (http://www.targetscan.org) predicted that Bcl-2 is the target gene of miR-503 conserved between different species. To determine whether Bcl-2 is the target gene of miR-503, we constructed a luciferase reporter vector with the putative Bcl-2 3'-UTR target site for miR-503 downstream of the luciferase gene (pGL3-Bcl-2-3'-UTR). The luciferase reporter vector together with the miR-503 mimic or the miRNA mimic control was transfected into the A549 and A549/CDDP cells. In both cell lines, a significant decrease in relative luciferase activity was observed when the pGL3-Bcl-2-3'-UTR vector was co-transfected with the miR-503 mimic but not with the
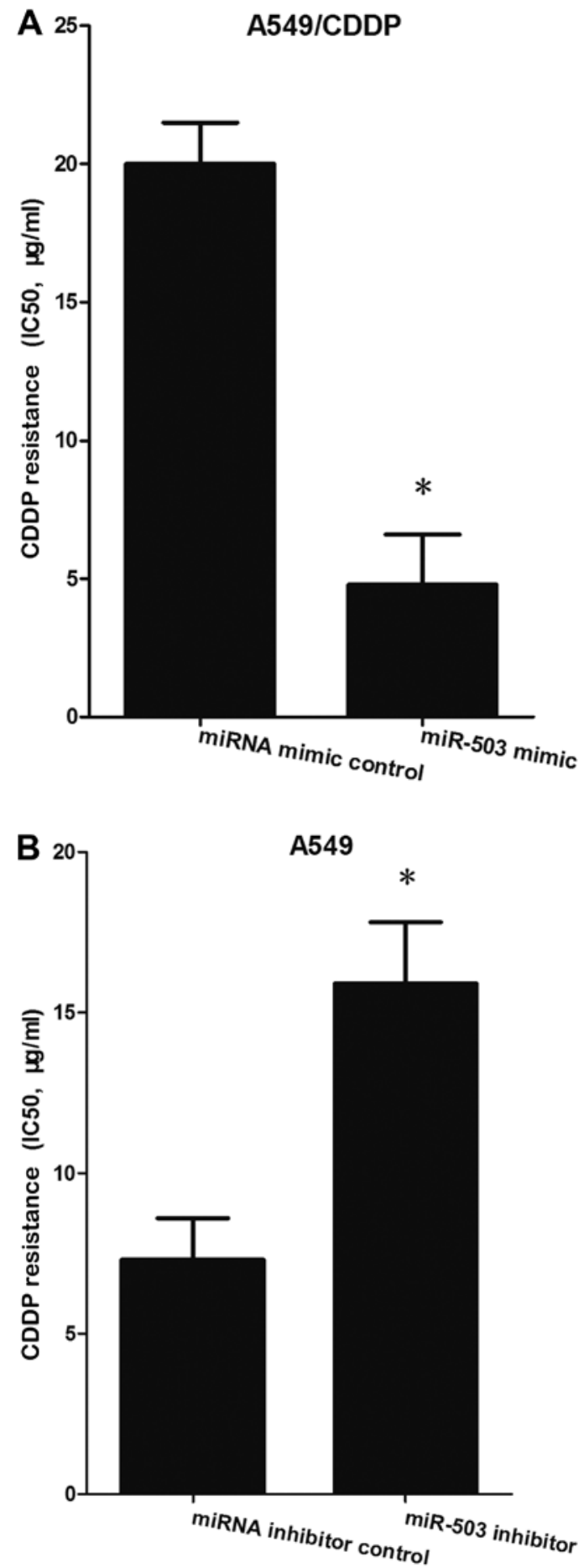

Figure 2. miR-503 sensitizes A549/CDDP cells to cisplatin (CDDP). (A) In the A549/CDDP cells, the cells transfected with the miR-503 mimic exhibited significantly decreased resistance to CDDP. (B) In the A549 cells, the cells transfected with the miR-503 inhibitor exhibited significantly enhanced resistance to CDDP. ${ }^{*} \mathrm{P}<0.01$.

miRNA mimic control, suggesting that $\mathrm{Bcl}-2$ is the target gene of miR-503 (Fig. 3).

miR-503 modulates resistance to cisplatin by suppressing Bcl-2 protein expression. A noteworthy observation was that the decreased expression of miR-503 in the A549/ CDDP cells was concurrent with the overexpression of Bcl-2 protein, compared with the parental A549 cells in our study 




Figure 3. Dual luciferase assay performed in A549 and A549/CDDP cells demonstrates that Bcl-2 is the target gene of miR-503. In the A549 and A549/ CDDP cells, a significant decrease in relative luciferase activity was noted when pGL3-Bcl-2-3'-UTR was co-transfected with the miR-503 mimic but not with the miRNA mimic control. ${ }^{*} \mathrm{P}<0.01$.

(Fig. 4A and C). Since the anti-apoptotic Bcl-2 protein is the target of miR-503, we hypothesized that miR-503 may modulate the drug resistance of cancer cells by suppressing Bcl-2 protein expression. To confirm our hypothesis, we transfected the miR-503 mimic and the control miRNA mimic into the A549/CDDP cells and detected changes in Bcl-2 expression levels. In the A549/CDDP cells, $72 \mathrm{~h}$ after transfection, western blot analysis revealed a significantly decreased Bcl-2 protein expression level in the miR-503 mimic-transfected cells compared with the miRNA mimic control-transfected cells (Fig. 4B and C). These results demonstrate that miR-503 modulates the resistance of cancer cells to cisplatin, at least in part by suppressing Bcl-2 protein expression.

miR-503 sensitizes A549/CDDP cells to CDDP-induced apoptosis. The development of drug resistance in various cancer cells has been linked to a reduced susceptibility to drug-induced apoptosis, which has been shown to be a consequence of the overexpression of anti-apoptotic proteins, such as Bcl-2 and $\mathrm{Bcl}-\mathrm{xL}(10,28,29)$. Since miR-503 regulates the drug resistance of cancer cells, at least in part by suppressing Bcl-2 protein expression, considering the well-characterized role of $\mathrm{Bcl}-2$ in apoptosis and drug resistance, we hypothesized that miR-503 plays a role in the development of drug resistance, at least in part through the modulation of apoptosis by targeting Bcl-2. To confirm this hypothesis, we evaluated cisplatin-induced apoptosis following the transfection of A549/CDDP cells with the miR-503 mimic or the miRNA mimic control. In the A549/ CDDP cells, a marked increase in apoptosis, as assessed by flow cytometry, was observed in the miR-503 mimic-transfected cells following treatment with cisplatin, compared with the miRNA mimic control-transfected cells (Fig. 5).

\section{Discussion}

Platinum-based drugs, cisplatin in particular, are the major clinical treatment drugs for non-small cell lung cancer at
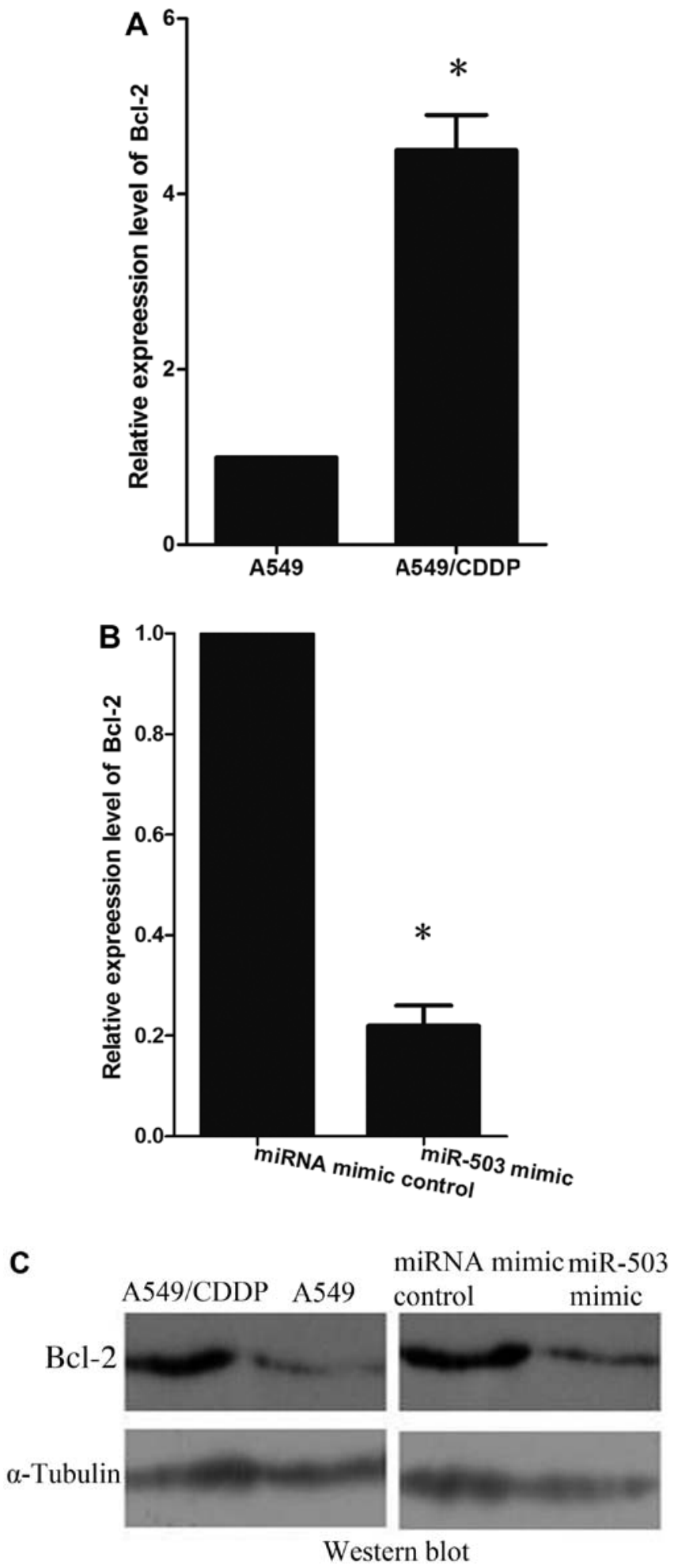

Figure 4. (A) Anti-apoptotic Bcl-2 protein is overexpressed in A549/CDDP cells compared with the parental A549 cells. This was shown by western blot analysis; representative image is shown on the top left panel in (C). (B) In the A549/CDDP cells, $72 \mathrm{~h}$ after transfection, western blot analysis demonstrated a significantly decreased Bcl-2 protein level in the miR-503 mimic-transfected cells compared with the miRNA mimic control-transfected cells. Representative image from western blot analysis is shown on the top right panel in (C). The results represent the means \pm SD from three independent experiments. ${ }^{*} \mathrm{P}<0.01$.

present. Despite tremendous efforts, cisplatin treatment often results in the development of drug resistance, leading to thera- 



Figure 5. (A) miR-503 mimic sensitized A549/CDDP cells to cisplatin (CDDP)-induced apoptosis. (B) In the A549/CDDP cells, apoptosis was evaluated by flow cytometry. The results revealed a marked increase in apoptosis in miR-503 mimic-transfected cells following treatment with CDDP, compared with the miRNA mimic control-transfected cells. Representative flow cytometry report is shown beside the graph. The results shown represent the means \pm SD from three independent experiments. ${ }^{*} \mathrm{P}<0.01$.

peutic failure. Therefore, cisplatin drug resistance has become an important clinical issue which needs to be resolved. It has been suggested that the mechanisms of cisplatin resistance involve reduced intracellular cisplatin accumulation, increased inactivation of cisplatin by thiol-containing molecules, enhanced DNA damage repair and the inhibition of transmitted DNA damage recognition to the apoptotic pathway (30). Among these, the acquired imbalance of apoptotic pathways is thought to be one of the most important mechanisms involved in drug resistance. Several apoptotic inhibitors have been associated with platinum resistance, including X-linked inhibitor of apoptosis (XIAP), Bcl-2 and Bcl-xL (31-34). Bcl-2 is an important survival factor which suppresses apoptosis in a variety of cell systems and regulates cell death by controlling the mitochondrial membrane permeability. The resistance of cancer cells to cisplatin may be, in some cases, caused by the overexpression of Bcl-2 (31). Consistent with this, in our study, we found that the anti-apoptotic protein, $\mathrm{Bcl}-2$, was upregulated in the cisplatin-resistant A549/CDDP cells compared with the A549 cells.

Since defective apoptosis may contribute to cisplatin drug resistance, miRNAs may modulate the drug sensitivity of cancer cells, at least in part through this mechanism $(14,24,35)$. Of note, miR-503 was downregulated in the A549/CDDP cells and the elevated levels of miR-503 not only downregulated the expression of Bcl-2 protein but also increased the sensitivity of these cells to cisplatin. The data presented in this study, provide evidence that miR-503 may be involved in the devel- opment of the resistance of non-small cell lung cancer cells to cisplatin by targeting Bcl- 2 .

miR-503 is located on chromosomal band Xq26.3, based on the sequence AGCAGC starting at the second nucleotide from the $5^{\prime}$ end of the mature ( $22 \mathrm{nt}$, single-stranded) miRNA, a motif which is referred to as AGC X2. As shown in previous studies, miR-503 is differentially expressed in different types of tumors, suggesting that the expression of miR-503 is tissuespecificity (16-20). In the present study, we found that miR-503 was downregulated in the cisplatin resistant non-small cell lung cancer cells. A previous study demonstrated that epigenetic alterations promote the dysregulation of miRNAs (36). Among these, the DNA methylation of $\mathrm{CpG}$ islands has been observed in the promoter region of miRNAs with tumor suppressor functions in human cancer, such as miRNAs miR-497, miR-127 and miR-1 (37-39). According to the data from the UCSC database (http://genome.ucsc.edu/cgi-bin/hgTracks?hgHub Connect.destUrl=..\%2Fcgi-bin $\% 2$ FhgTracks\&clade $=$ mammal \&org=Human\&db=hg19\&position=miR-503\&hgt.positionInput $=$ miR-503\&hgt.suggestTrack $=$ knownGene $\&$ Submit $=$ submit $\&$ hgsid=298724897), the DNA methylation of CpG islands in the promoter region may lead to the downregulation of miR-503 in drug-resistant cells. However, further studies are required to elucidate the underlying mechanisms.

In conclusion, the data presented in this study provide evidence of the involvement of miR-503 in the development of the resistance of human non-small cell lung cancer cells to cisplatin. Hsa-miR-503 sensitizes human drug-resistant 
lung cancer cells to cisplatin, at least in part by targeting Bcl-2 expression. Our findings may contribute to the further understanding of the regulation of drug resistance in cancer cells. Therapeutic strategies targeting the miRNAs associated with drug resistance, such as hsa-miR-503, may prove to be a promising approach for the development of safe and effective clinical treatments. However, it should be noted that our data were derived from cell lines which have been removed from their in vivo context and cannot be considered accurate surrogates for clinical tumors. Thus, further clinical studies are warranted to assess the role of hsa-miR-503 in vivo.

\section{Acknowledgements}

The authors are grateful for the funding provided by the National Natural Science Foundation of China (grant nos. 81171908 and 81201705) and the Research and Innovation Project for College Graduates of Jiangsu Province (grant no. JX22013193).

\section{References}

1. Szakács G, Paterson JK, Ludwig JA, et al: Targeting multidrug resistance in cancer. Nat Rev Drug Discov 5: 219-234, 2006.

2. Zhang K, Mack P and Wong KP: Glutathione-related mechanisms in cellular resistance to anticancer drugs. Int J Oncol 12: 871-882, 1998

3. Johnstone RW, Ruefli AA and Lowe SW: Apoptosis: a link between cancer genetics and chemotherapy. Cell 108: 153-164, 2002.

4. Rabik CA and Dolan ME: Molecular mechanisms of resistance and toxicity associated with platinating agents. Cancer Treat Rev 33: 9-23, 2007.

5. Fojo T: Multiple paths to a drug resistance phenotype: mutations, translocations, deletions and amplification of coding genes or promoter regions, epigenetic changes and microRNAs. Drug Resist Updat 10: 59-67, 2007.

6. Glasspool RM, Teodoridis JM and Brown R: Epigenetics as a mechanism driving polygenic clinical drug resistance. Br J Cancer 94: 1087-1092, 2006.

7. Sharma SV, Lee DY, Li B, et al: A chromatin-mediated reversible drug-tolerant state in cancer cell subpopulations. Cell 141: 69-80, 2010.

8. Lee RC,Feinbaum RL and Ambros V: The C.elegans heterochronic gene lin-4 encodes small RNAs with antisense complementarity to lin-14. Cell 75: 843-854, 1993.

9. Garzon R, Marcucci G and Croce CM: Targeting microRNAs in cancer: rationale, strategies and challenges. Nat Rev Drug Discov 9: 775-789, 2010.

10. Chen GQ, Zhao ZW, Zhou HY, et al: Systematic analysis of microRNA involved in resistance of the MCF-7 human breast cancer cell to doxorubicin. Med Oncol 27: 406-415, 2010.

11. Bourguignon LY, Spevak CC, Wong G, et al: Hyaluronan-CD44 interaction with protein kinase $\mathrm{C}$ (epsilon) promotes oncogenic signaling by the stem cell marker Nanog and the production of microRNA-21, leading to down-regulation of the tumor suppressor protein PDCD4, anti-apoptosis, and chemotherapy resistance in breast tumor cells. J Biol Chem 284: 26533-26546, 2009.

12. Li Y, Li W, Yang Y, et al: MicroRNA-21 targets LRRFIP1 and contributes to VM-26 resistance in glioblastoma multiforme. Brain Res 1286: 13-18, 2009.

13. Xu K, Liang X, Cui D, et al: miR-1915 inhibits Bcl-2 to modulate multidrug resistance by increasing drug-sensitivity in human colorectal carcinoma cells. Mol Carcinog 52: 70-78, 2013.

14. Xia L, Zhang D, Du R, et al: miR-15b and miR-16 modulate multidrug resistance by targeting BCL2 in human gastric cancer cells. Int J Cancer 123: 372-379, 2008

15. Chen J, Tian W, Cai H, et al: Down-regulation of microRNA-200c is associated with drug resistance in human breast cancer. Med Oncol 29: 2527-2534, 2012.
16. Zhou J and Wang W: Analysis of microRNA expression profiling identifies microRNA-503 regulates metastatic function in hepatocellular cancer cell. J Surg Oncol 104: 278-283, 2011.

17. Lu YC, Chen YJ, Wang HM, et al: Oncogenic function and early detection potential of miRNA-10b in oral cancer as identified by microRNA profiling. Cancer Prev Res (Phila) 5: 665-674, 2012.

18. Özata DM, Caramuta S, Velázquez-Fernández D, et al: The role of microRNA deregulation in the pathogenesis of adrenocortical carcinoma. Endocr Relat Cancer 18: 643-655, 2011.

19. Corbetta S, Vaira V, Guarnieri V, et al: Differential expression of microRNAs in human parathyroid carcinomas compared with normal parathyroid tissue. Endocr Relat Cancer 17: 135-146, 2010.

20. Zhao JJ, Yang J, Lin J, et al: Identification of miRNAs associated with tumorigenesis of retinoblastoma by miRNA microarray analysis. Childs Nerv Syst 25: 13-20, 2009.

21. Cui YH, Xiao L, Rao JN, et al: miR-503 represses CUG-binding protein 1 translation by recruiting CUGBP1 mRNA to processing bodies. Mol Biol Cell 23: 151-162, 2012.

22. Jiang Q, Feng MG and Mo YY: Systematic validation of predicted microRNAs for cyclin D1. BMC Cancer 9: 194, 2009.

23. Cheng G, Sun S, Wang Z and Jin S: Investigation of the interaction between the miR-503 and CD40 genes in irradiated U937 cells. Radiat Oncol 7: 38, 2012.

24. Zhu W, Zhu D, Lu S, et al: miR-497 modulates multidrug resistance of human cancer cell lines by targeting BCL2. Med Oncol 29: 384-391, 2012.

25. Livak KJ and Schmittgen TD: Analysis of relative gene expression data using real-time quantitative PCR and the 2(-Delta Delta C(T)) method. Methods 25: 402-408, 2001.

26. Yamaue $\mathrm{H}$, Tanimura $\mathrm{H}$, Noguchi $\mathrm{K}$, et al: Chemosensitivity testing of fresh human gastric cancer with highly purified tumor cells using the MTT assay. Br J Cancer 66: 794-799, 1992.

27. Yamaue $\mathrm{H}$, Tani $\mathrm{M}$, Onishi $\mathrm{H}$, et al: Locoregional chemotherapy for patients with pancreatic cancer intra-arterial adjuvant chemotherapy after pancreatectomy with portal vein resection. Pancreas 25: 366-372, 2002.

28. Wang S, Yang D and Lippman ME: Targeting Bcl-2 and Bcl-XL with nonpeptidic small-molecule antagonists. Semin Oncol 30 (Suppl 16): S133-S142, 2003.

29. Reed JC: Drug insight: cancer therapy strategies based on restoration of endogenous cell death mechanisms. Nat Clin Prac Oncol 3: 388-398, 2006

30. Tanida S, Mizoshita T, Ozeki K, et al: Mechanisms of cisplatininduced apoptosis and of cisplatin sensitivity: potential of BIN1 to act as a potent predictor of cisplatin sensitivity in gastric cancer treatment. Int J Surg Oncol 2012: 862879, 2012.

31. Beale PJ, Rogers P, Boxall F, et al: BCL-2 family protein expression and platinum drug resistance in ovarian carcinoma. $\mathrm{Br} \mathrm{J}$ Cancer 82: 436-440, 2000.

32. Yu L and Wang Z: Difference in expression of Bcl-2 and Bcl-xl genes in cisplatin-sensitive and cisplatin-resistant human in ovarian cancer cell lines. J Huazhong Univ Sci Technolog Med Sci 24: 151-153, 2004.

33. Dong $\mathrm{Z}$ and Wang J: Hypoxia selection of death-resistant cells. A role for Bcl-X(L). J Biol Chem 279: 9215-9221, 2004.

34. Williams J, Lucas PC, Griffith KA, et al: Expression of Bcl-xL in ovarian carcinoma is associated with chemoresistance and recurrent disease. Gynecol Oncol 96: 287-295, 2005.

35. Zhu W, Shan X, Wang TS, et al: miR-181b modulates multidrug resistance by targeting BCL2 in human cancer cell lines. Int $\mathrm{J}$ Cancer 127: 2520-2529, 2010.

36. Baylin SB: DNA methylation and gene silencing in cancer. Nat Clin Pract Oncol 2 (Suppl 1): S4-S11, 2005.

37. Li D, Zhao Y, Liu C, et al: Analysis of MiR-195 and MiR-497 expression, regulation and role in breast cancer. Clin Cancer Res17: 1722-1730, 2011.

38. Saito Y, Liang G, Egger G, et al: Specific activation of microRNA-127 with downregulation of the proto-oncogene BCL6 by chromatin-modifying drugs in human cancer cells. Cancer Cell 9: 435-443, 2006.

39. Datta J, Kutay H, Nasser MW, et al: Methylation mediated silencing of MicroRNA-1 gene and its role in hepatocellular carcinogenesis. Cancer Res 68: 5049-5058, 2008. 